\title{
Acetaminophen metabolism revisited using non-targeted analyses Implications for human biomonitoring
}

David, Arthur; Chaker, Jade; Léger, Thibaut; AI-Salhi, Raghad; Dalgaard, Marlene D.; Styrishave, Bjarne; Bury, Daniel; Koch, Holger M.; Jégou, Bernard; Kristensen, David M.

\section{Published in:}

Environment International

Link to article, DOI:

10.1016/j.envint.2021.106388

Publication date:

2021

Document Version

Publisher's PDF, also known as Version of record

Link back to DTU Orbit

Citation (APA):

David, A., Chaker, J., Léger, T., Al-Salhi, R., Dalgaard, M. D., Styrishave, B., Bury, D., Koch, H. M., Jégou, B., \& Kristensen, D. M. (2021). Acetaminophen metabolism revisited using non-targeted analyses: Implications for human biomonitoring. Environment International, 149, [106388]. https://doi.org/10.1016/j.envint.2021.106388

\section{General rights}

Copyright and moral rights for the publications made accessible in the public portal are retained by the authors and/or other copyright owners and it is a condition of accessing publications that users recognise and abide by the legal requirements associated with these rights.

- Users may download and print one copy of any publication from the public portal for the purpose of private study or research.

- You may not further distribute the material or use it for any profit-making activity or commercial gain

- You may freely distribute the URL identifying the publication in the public portal 


\title{
Acetaminophen metabolism revisited using non-targeted analyses: Implications for human biomonitoring
}

\author{
Arthur David $^{\text {a, },}$, Jade Chaker ${ }^{\mathrm{a}}$, Thibaut Léger ${ }^{\mathrm{a}}$, Raghad Al-Salhi ${ }^{\mathrm{a}}$, Marlene D. Dalgaard ${ }^{\mathrm{b}}$, \\ Bjarne Styrishave $^{c}$, Daniel Bury ${ }^{\mathrm{d}}$, Holger M. Koch ${ }^{\mathrm{d}}$, Bernard Jégou ${ }^{\mathrm{a}, 1}$, David M. Kristensen ${ }^{\text {a, e, } 1}$ \\ a Univ Rennes, Inserm, EHESP, Irset (Institut de recherche en santé, environnement et travail) - UMR_S 1085, F-35000 Rennes, France \\ ${ }^{\mathrm{b}}$ Department of Health Technology, Technical University of Denmark, Denmark \\ ${ }^{\mathrm{c}}$ Department of Pharmacy, Faculty of Health and Medical Sciences, University of Copenhagen, Denmark \\ ${ }^{\mathrm{d}}$ Institute for Prevention and Occupational Medicine of the German Social Accident Insurance - Institute of the Ruhr-University Bochum (IPA), Bürkle-de-la-Camp-Platz \\ 1, 44789 Bochum, Germany \\ e Danish Headache Center, Department of Neurology, Rigshospitalet-Glostrup, Denmark
}

\section{A R T I C L E I N F O}

Handling editor: Heather Stapleton

\section{Keywords:}

Paracetamol/acetaminophen

Human biomonitoring

Non-targeted analyses

High-resolution mass spectrometry (HRMS)

\begin{abstract}
A B S T R A C T
The analgesic paracetamol/acetaminophen (N-acetyl-4-aminophenol, APAP) is commonly used to relieve pain, fever and malaise. While sales have increased worldwide, a growing body of experimental and epidemiological evidence has suggested APAP as a possible risk factor for various health disorders in humans. To perform internal exposure-based risk assessment, the use of accurate and optimized biomonitoring methods is critical. However, retrospectively assessing pharmaceutical use of APAP in humans is challenging because of its short half-life. The objective of this study was to address the key issue of potential underestimation of APAP use using current standard analytical methods based on urinary analyses of free APAP and its phase II conjugates. The question we address is whether investigating additional metabolites than direct phase II conjugates could improve the monitoring of APAP. Using non-targeted analyses based on high-resolution mass spectrometry, we identified, in a controlled longitudinal exposure study with male volunteers, overlooked APAP metabolites with delayed formation and excretion rates. We postulate that these metabolites are formed via the thiomethyl shunt after the enterohepatic circulation as already observed in rodents. Importantly, these conjugated thiomethyl metabolites were (i) of comparable diagnostic sensitivity as the free APAP and its phase II conjugates detected by current methods; (ii) had delayed peak levels in blood and urine compared to other APAP metabolites and therefore potentially extend the window of exposure assessment; and (iii) provide relevant information regarding metabolic pathways of interest from a toxicological point of view. Including these metabolites in future APAP biomonitoring methods therefore provides an option to decrease potential underestimation of APAP use. Moreover, our data challenge the notion that the standard methods in biomonitoring based exclusively on the parent compound and its phase II metabolites are adequate for human biomonitoring of a non-persistent chemical such as APAP.
\end{abstract}

Abbreviations: APAP, acetaminophen; APAP-S, APAP sulfate; APAP-Glu, APAP glucuronide; NAPQI, N-acetyl-p-benzoquinone imine; 3-OH-APAP, 3-hydrox-

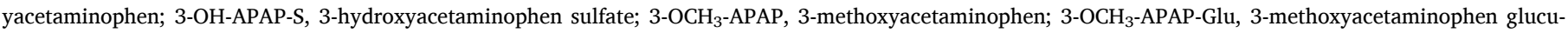

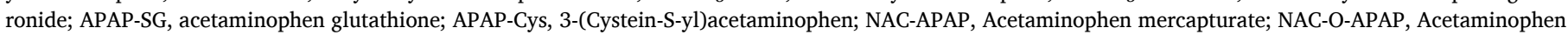

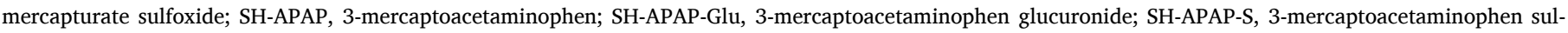

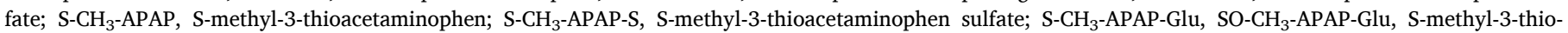
acetaminophen glucuronide; SO- $\mathrm{CH}_{3}$-APAP, S-methyl-3-thioacetaminophen sulphoxide; $\mathrm{SO}_{-} \mathrm{CH}_{3}$-APAP-S, S-methyl-3-thioacetaminophen sulphoxide sulphate.

* Corresponding author.

E-mail address: arthur.david@ehesp.fr (A. David).

1 Joint last authors. 


\section{Introduction}

The mild analgesic paracetamol/acetaminophen ( $\mathrm{N}$-acetyl-4-aminophenol, APAP) is an active ingredient in more than 600 prescription and non-prescription pharmaceuticals (Hurwitz et al., 2014). This analgesic is one of the most commonly used pharmaceuticals worldwide, with sales that have steadily increased over the past 20 years in several countries (Kristensen et al., 2016). The medical benefits of APAP are widely recognized; APAP can be used on its own, in mixed formulations or as an alternative to non-steroidal anti-inflammatory drugs whose suspected negative side effects have raised concerns (Kristensen et al., 2018). However, recent experimental as well as epidemiological studies suggest APAP as a possible risk factor for male developmental disorders in humans, on its own, or in combination with other reproductive and developmental toxicants (Albert et al., 2013; Konkel, 2018; Kortenkamp, 2020; Kortenkamp and Koch, 2020; Kristensen et al., 2012; Kristensen et al., 2016). Furthermore, incorrect use of APAP is strongly associated with a broad spectrum of side effects and is the most common cause of acute liver failure (fulminant liver failure) with over 100,000 cases alone in the US (Ferri, 2016).

Besides APAP exposure through intentional pharmaceutical use, it has been shown that APAP is ubiquitously excreted in the urine of nonusers in the general population (medians of $61.7 \mu \mathrm{g} / \mathrm{L}$ and $100 \mu \mathrm{g} / \mathrm{L}$ from the German (men and women) (Modick et al., 2014) and Danish (women and children) (Nielsen et al., 2015) populations, respectively), indicating that an unintentional environmental exposure exists. The environmental exposure has been linked to APAP precursors such as aniline and 4-aminophenol (two large-volume intermediates in industrial processes) and/or indirect APAP exposure through environmental sources (Kristensen et al., 2016; Modick et al., 2014; Modick et al., 2016). The APAP urinary background concentrations measured in non-users are about 4000 fold lower than the maximum urinary concentrations measured after oral intake of $500 \mathrm{mg}$ APAP, but in the range of concentrations measured $24-48 \mathrm{~h}$ after such an intentional oral intake (Modick et al., 2014). Hence, these environmental APAP urinary concentrations can interfere when assessing APAP use in epidemiological studies especially if intentional APAP intake occurred $>24 \mathrm{~h}$ before sampling.

Understanding the amount of a chemical that enters the human body, whether from intentional use or unintentional exposure, is essential for both exposure and risk assessment. APAP metabolism is rapid (half-life $<3 \mathrm{~h}$ in blood (Mazaleuskaya et al., 2015)) and the large majority of the APAP therapeutic dose is classically believed to be excreted in urine as direct phase II glucuronide and sulfate conjugates. Mercapturic acid and cysteine conjugates derived from glutathione conjugation of the reactive metabolite N-acetyl-p-benzoquinone imine (NAPQI) are also produced in APAP metabolism. However, these metabolites are generally considered as minor metabolites together with catechol metabolites produced by CYP450 enzymes (Bessems and Vermeulen, 2001; Mazaleuskaya et al., 2015). Thus, present standard targeted methods analyzing free APAP and the phase II conjugates as "total APAP" after enzymatic deconjugation have been considered as the valid standard methods for estimating internal exposure for risk assessment purposes (Bornehag et al., 2018; Modick et al., 2014; Nielsen et al., 2015). However, these methods capture only part of the metabolic pathway and a relatively short time window to assess APAP origin (i.e. pharmaceutical use versus environmental), leading to potential underestimations of actual exposures. Exposure underestimations may have large implications as they could fundamentally compromise outcomes of risk assessment decisions.

In this study, we address the key issue of potential underestimation of APAP use using current human-biomonitoring methods focused on free APAP and phase II conjugates, only. We monitored APAP exposure over four days in blood and urine of four male volunteers with an administration of $1 \mathrm{~g}$ of APAP on the third day. Using recent untargeted technological advancements of high-resolution mass spectrometry (HRMS) combined with omics-based data treatment, we investigated the presence of neglected APAP metabolites from the enterohepatic circulation with formation and excretion times potentially longer than APAP phase II metabolites. Such additional knowledge can improve the human biomonitoring approach for the non-persistent chemical APAP in future exposure and risk assessment studies.

\section{Materials and methods}

\subsection{Longitudinal clinical trial}

\subsubsection{Design and participants}

The human in vivo study ( $\mathrm{n}=4$ ) was designed as a longitudinal exposure to APAP over four days with an administration of $1 \mathrm{~g}$ of APAP on the third day (Fig. S1). The study protocol was in compliance with the Helsinki Declaration and was approved by the Regional Scientific Ethical Committees of Copenhagen in Denmark (Protocol nr.: 17003845; and as part of trial H-17002476). The study recruited 4 healthy men aged 30-60 years at the Department of Pharmacy and Department of Biology, University of Copenhagen. Exclusion criteria include other use of medicine and body mass index above 30, peptic ulcers, signs of liver or kidney dysfunction. All individuals provided oral informed consent to participate in the study.

\subsubsection{Collection of blood plasma and urine samples before and after APAP intake}

The subjects received APAP $2 \times 500 \mathrm{mg}$ (Panodil ${ }^{\circledR}$, GlaxoSmithKline Aps). No subjects reported any adverse signs of taking the medication. Three baseline samples of urine (spot urine) and plasma were sampled in the morning two days and just prior to intake of APAP. Subsequently, samples were taken $+1 \mathrm{~h},+2 \mathrm{~h}$ (urine only), $+4 \mathrm{~h},+6 \mathrm{~h}$ hours after intake and in the morning of the subsequent two days ( +24 and $48 \mathrm{~h}$ ) (Fig. S1.). For each time point, 10-mL heparinized blood samples were collected from the antecubital vein of the non-dominant arm together with urine samples. Blood samples were centrifuged (5 min, 10,000g) and plasma collected. Plasma and urine samples were stored at $-80{ }^{\circ} \mathrm{C}$ immediately after collection.

\subsubsection{Targeted analyses of APAP in blood plasma and urine}

APAP was analyzed using an adapted version of a method previously described (Modick et al., 2013; Modick et al., 2016). In an HPLC vial, $300 \mu \mathrm{L}$ ammonium acetate buffer (0.5 M, pH 5.5-6.0), $6 \mu \mathrm{L} \beta$-glucuronidase type HP-2 from Helix pomatia (with aryl sulfatase activity; Sigma Aldrich, Steinheim, Germany), and $30 \mu \mathrm{L}$ internal standard (1 $\mathrm{mg} / \mathrm{L} N$-(4-hydroxyphenyl-2,3,5,6- $d_{4}$ )acetamide (APAP- $d_{4}$ ) in water) were added to $300 \mu \mathrm{L}$ of the sample. After incubation at $37^{\circ} \mathrm{C}$ for $3.5 \mathrm{~h}$, $160 \mu \mathrm{L} 3 \mathrm{M}$ formic acid were added and the samples were frozen overnight. The thawed and homogenized samples were centrifuged (10 min, $1900 \mathrm{~g}$ ) and the supernatant analyzed by online-SPE-LC-ESI-MS/MS. A 1260 Infinity HPLC (Agilent Technologies, Waldbronn, Germany) consisting of a G1367E autosampler with G1330B thermostat, a G1312B binary pump (LC pump) with G4225A degasser, a G1311B quaternary low pressure gradient pump (loading pump) and a G1316A thermostated column compartment with a six-port switching valve was used for the LC separation (setup depicted in Modick et al., 2013) and a 4500 triple quadrupole mass spectrometer (Sciex, Darmstadt, Germany) was used for detection. An Oasis HLB cartridge $(2.1 \times 20 \mathrm{~mm} ; 25 \mu \mathrm{m}$; Waters, Eschborn, Germany) was used for online SPE and an Accucore Phenyl-X $(3.0 \times 150 \mathrm{~mm} ; 2.6 \mu \mathrm{m}$; Thermo Scientific, Franklin, MA, USA $)$ was used for the HPLC separation. Eluents were water (A) and acetonitrile (B), both containing $0.05 \%$ formic acid. $25 \mu \mathrm{L}$ of the sample were loaded onto the SPE column at a flow of $3.75 \mathrm{~mL} / \mathrm{min}$ pure eluent A. The sixport valve was switched at $\mathrm{t}=2 \mathrm{~min}$ to start the analyte transfer from the SPE column onto the analytical column (in back-flush) and was switched back to the loading position at $\mathrm{t}=4.5 \mathrm{~min}$. From $\mathrm{t}=4.5 \mathrm{~min}$ to $16 \mathrm{~min}$, the SPE column was flushed with $95 \%$ B for 5 min and then reequilibrated to the starting conditions simultaneous to the analytical 
separation. The gradient for the analytical separation started at $15 \%$ solvent B. After $0.5 \mathrm{~min}$, B was increased to $35 \%$ within $0.5 \mathrm{~min}$ and then increased to $45 \%$ within $3.5 \mathrm{~min}$. Afterwards, B was increased to $50 \%$ within $1.5 \mathrm{~min}$ and then to $95 \%$ within $2 \mathrm{~min}$ and kept for $2.5 \mathrm{~min}$. B was then returned to initial 15\% within $2.5 \mathrm{~min}$ and kept for $3 \mathrm{~min}$. Detection of APAP and APAP- $d_{4}$ was performed with ESI-MS in positive ionization mode (time-programmed MRM). Ion source parameters were (all gases nitrogen) 35 psi curtain gas, 25 psi nebulizer and heater gas, $4.5 \mathrm{kV}$ electrospray voltage, and $450{ }^{\circ} \mathrm{C}$ source temperature. Quantifier mass transitions for APAP $\left(t_{R}=6 \mathrm{~min}\right)$ and APAP- $d_{4}\left(t_{R}=6 \mathrm{~min}\right)$ were $m / z$ $152 \rightarrow 110$ and $m / z 156 \rightarrow 114$ (collision energy (CE) $22 \mathrm{eV}$, collision cell exit potential (CXP) 7.7 V), respectively. Qualifier mass transitions were $m / z 152 \rightarrow 93$ and $m / z 156 \rightarrow 97$ (CE $28 \mathrm{eV}$, CXP $6.3 \mathrm{~V}$ ), respectively. The collision gas pressure setting was 5 arbitrary units and the entrance potential and declustering potential were $12 \mathrm{~V}$ and $59 \mathrm{~V}$, respectively. The limit of quantification (based on a signal-to-noise ratio of 10) was $1 \mu \mathrm{g} / \mathrm{L}$ APAP. Plasma samples were analyzed at least in 2-fold dilution (diluted with water). Urine concentrations were normalized to specific gravity measured using a refractometer (Atago Urine S.G. scale: 1.000 to 1.060 ).

\subsubsection{Non-targeted analyses of APAP in blood plasma and urine}

2.1.4.1. Chemicals. The list of standards used for the identification of APAP metabolites and the 15 labelled internal standards (IS) spiked in samples for the untargeted analyses and their respective suppliers are provided in Table S1. All solvents were high-performance liquid chromatography grade, purchased from Biosolve Chime (Dieuze, France). Phree Phospholipid Removal 96-well plates and Strata-X Polymeric Reversed Phase cartridges (200 mg, $3 \mathrm{~mL}$ ) were supplied by Phenomenex (Le Pecq, France).

2.1.4.2. Preparation of blood plasma and urine samples. Plasma samples were prepared based on the method published in David et al. (2014). Previous studies have demonstrated that this sample preparation method developed for untargeted analysis purposes using phospholipid removal followed by polymeric reversed phase solid phase extraction extracts xenobiotics such as pharmaceuticals (including APAP), conjugated xenobiotics as well as non-polar, cationic and anionic metabolites (e.g., steroids, eicosanoids, amino acids, neurotransmitters, bile acids and lipids) from plasma samples (David et al., 2014; David et al., 2017). Briefly, $400 \mu \mathrm{L}$ of acetonitrile with $1 \%$ formic acid (FA) was added to each blood plasma sample $(100 \mu \mathrm{L})$. The IS mixture containing $1.5 \mathrm{ng}$ of each labelled standard (see Table S1) was added to the plasma extracts. After homogenization, samples were filtered under vacuum using a 96well sample manifold and the filtrate collected. An additional $100 \mu \mathrm{L}$ of acetonitrile containing 1\% FA was added to the wells at the end of the filtration process to rinse the sorbent. The combined filtrates were collected and $4 \mathrm{~mL}$ of HPLC grade water was added to each sample. Samples were homogenized and then extracted using Strata-X cartridges which were conditioned with $4 \mathrm{~mL}$ methanol and equilibrated with $4 \mathrm{~mL}$ HPLC grade water. Samples were loaded onto the SPE cartridges. After a washing step using $2 \times 3 \mathrm{~mL}$ of 95/5 HPLC grade water/methanol (v/v), sorbents were dried under vacuum and elution was performed with $4 \mathrm{~mL}$ of methanol. The eluate was evaporated to dryness, reconstituted in 15 $\mu \mathrm{L} 80 / 20$ water/acetonitrile (v/v) and stored at $-80^{\circ} \mathrm{C}$ until analysis.

Urine samples were prepared using a similar SPE protocol as the one used for the plasma. The step before SPE, i.e. removal of phospholipid and protein using the Phree plates, was omitted as urine contains lower levels of these molecules (as opposed to plasma). Previous studies have shown that methods using polymeric SPE are efficient to concentrate urine samples for untargeted analyses and extract the urinary metabolome and (xeno)metabolome (Chetwynd et al., 2015). Briefly, urine samples $(500 \mu \mathrm{L})$ were diluted to $4 \mathrm{~mL}$ of HPLC grade water, acidified with $1 \%$ formic acid and extracted using Strata-X SPE. Cartridges were conditioned with $4 \mathrm{~mL}$ methanol and $4 \mathrm{~mL}$ of HPLC grade water. Strata$\mathrm{X}$ cartridges were then loaded with the $4 \mathrm{~mL}$ of diluted urine and washed with $2 \times 3 \mathrm{~mL}$ of 95/5 HPLC grade water/methanol (v/v). SPE phases were dried under vacuum and elution was performed with $4 \mathrm{~mL}$ of methanol. The eluate was evaporated to dryness, reconstituted in $50 \mu \mathrm{L}$ $80 / 20$ water/acetonitrile (v/v) and stored at $-80{ }^{\circ} \mathrm{C}$ until analysis.

2.1.4.3. UHPLC-ESI-QTOFMS analyses. Plasma and urine extracts were profiled using an Exon UHPLC system (AB Sciex, USA) coupled to an AB Sciex X500R Q-TOF-MS system (Sciex technologies, Canada), equipped with a DuoSpray ion source. $2 \mu \mathrm{L}$ of extracts were loaded and separated on an Acquity UHPLC HSS-T3 column, $1.0 \mathrm{~mm} \times 150 \mathrm{~mm} \times 1.8 \mu \mathrm{m}$, maintained at $40{ }^{\circ} \mathrm{C}$ (Waters Technologies, Saint Quentin, France). HPLC grade water was used as solvent A and acetonitrile as solvent B, both modified with $0.01 \%$ formic acid. The flow rate was $100 \mathrm{uL} / \mathrm{min}$ with a gradient of $0-2.5 \mathrm{~min}$ from $10 \%$ to $20 \% \mathrm{~B}, 2.5-20 \mathrm{~min}$ from 20 to $30 \% \mathrm{~B}, 20-38 \mathrm{~min}$ from $30 \%$ to $45 \% \mathrm{~B}, 38-45$ min from 45 to $100 \% \mathrm{~B}$, 45-55 $\min 100 \% \mathrm{~B}$, and equilibration to initial conditions in $5 \mathrm{~min}$.

The Q-TOF was recalibrated automatically after each sample using an ESI positive/negative calibration solution via a calibration delivery system (CDS). As a first step, all the samples were analysed in full scan experiment (50-1100 Da) in both - and + ESI modes. The common parameters for the full scan experiment in both positive/negative ion modes were: collision energy, $10 \mathrm{eV}$; curtain gas, 35 ; ion source gas 1 , 50 ; ion source gas 2,70 ; temperature, $550{ }^{\circ} \mathrm{C}$; declustering potential, 80 $\mathrm{V}$; mass resolution of 50,000.

As a second step, MS/MS mass fragmentation information for chemical elucidation was obtained by further analysis of selected samples in sequential window acquisition of theoretical mass spectrum (SWATH). Selection of samples were made based on the presence of markers discriminating between the 3 groups (baseline group, 1-6 h group and $+1-2$ days group, see HRMS dataset section) that needed further structural MSMS confirmation. Swath data-independent acquisitions were performed in order to achieve comprehensive MS/MS sampling. The Q1 isolation window strategy was generated using the Sciex Variable Window Table from previously acquired MS data on the specific sample of interest in order to optimize window widths across the entire $\mathrm{m} / \mathrm{z}$ range. SWATH experiments were performed in both - and + mode with parameters: MS1 accumulation time, $80 \mathrm{~ms}$; MS2 accumulation time, $30 \mathrm{~ms}$; collision energy, $35 \mathrm{eV}$; collision energy spread, $15 \mathrm{eV}$; cycle time, $469 \mathrm{~ms}$; mass range, $\mathrm{m} / \mathrm{z}$ 50-900.

2.1.4.4. Quality control. For the untargeted analysis, one workup blank sample (i.e., extraction with HPLC grade water instead of sample) per analytical batch was prepared to monitor for background contaminants. Quality control (QC) samples comprising a composite sample were prepared in order to monitor for UHPLC-ESI-TOF-MS repeatability and sensitivity during analysis of a sample run. Solvent blank samples (acetonitrile $/ \mathrm{H}_{2} \mathrm{O}(20: 80)$ ) were also injected to ensure that there was no carryover in the UHPLC system that might affect adjacent results in analytical runs. Each batch commenced with the injection of the blank samples (workup and solvent) followed by the injection of a QC sample, and then all samples from the batch were injected randomly. As recommended, QC samples were injected within each batch periodically (i. e. every 7 sample for plasma and 5 sample for urine) to monitor the analytical sensitivity and the repeatability ( $n=6$ QCs in plasma and $n=$ 9 QCs in urine, for each mode). To assess the UHPLC-ESI-Q-TOF-MS analytical variability during analytical runs, coefficients of variation (CV) for the peak areas were calculated for each IS in QC samples. Furthermore, the analytical variability of the plasma and urine runs was also assessed based upon the method proposed by Want et al. (2010). The response (i.e., the area) of each marker (Rt $\times m / z$ values) from all $\mathrm{QC}$ samples was normalized to the total ion signal (area) to monitor the analytical variability at the whole metabolome scale. Using this method, mean area of more than $80 \%$ of all markers present in QCs had CVs of 
less than $30 \%$ as recommended by Want et al. (2010) for metabolomics experiments (Table S4). Coefficients of variation (CVs) of the peak areas of each labelled internal standard $(n=15)$ measured in quality controls (QCs) in plasma $(\mathrm{n}=6 \mathrm{QCs})$ and urine $(\mathrm{n}=9 \mathrm{QCs})$ were all below $20 \%$ (Table S5). To correct for extraction issues and matrix interferences, the peak area of each metabolite was adjusted to that of APAP-d4 in case of blood and to the total peak area (or sum of peak areas) in urine (Gagnebin et al., 2017) to correct mainly for urinary dilution issues.

2.1.4.5. HRMS dataset. Mass spectra collected in full scan mode $(\mathrm{m} / \mathrm{z}$ 50-1100) and spectral peaks were preprocessed (i.e., deconvolution, alignment, peak picking) using both the open source R package XCMS (Smith et al., 2006) and vendor software MarkerView 1.3.1 (AB, Sciex) to create separate aggregate datasets from all sample files in which the MS features were binned into retention time (Rt) $\times m / z$ values. For XCMS, the raw data files were first converted to 64 bit .mzML (full scan) using MSConvert from ProteoWizard (Adusumilli and Mallick, 2017).

The peak tables obtained with XCMS were used for multivariate and univariate analyses in an $R$ environment ( $R$ version 3.6.0) in order to identify APAP metabolites after APAP exposure (see Table S2 for parameters used). Principal component analyses (PCA) were performed as a first step to examine QC clustering, sample separation and identify any analytical or biological outliers (See Fig. S2 and S3). Sample groups were then defined as follows in order to perform Sparse Partial Least Squares Discriminant Analyses (sPLS-DA) models: baseline group = samples collected two days and just prior to intake of APAP, 1-6 h group = samples collected $+1,+2,+4,+6 \mathrm{~h}$ after intake and $+1-2$ days group $=$ samples collected in the morning the subsequent two days. sPLS-DA was performed with mixOmics $\mathrm{R}$ package (version 6.8.5) in the mode regression (Rohart et al., 2017) (See Fig. S4 and S5). Besides multivariate analyses, univariate analyses were performed to compare fold changes of markers between the three different groups with the mixOmics $\mathrm{R}$ package. A corresponding $\mathrm{p}$-value was calculated with the multtest R package using Unpaired Student's t-tests, with an Adaptive Benjamini and Hochberg ( $\mathrm{ABH}$ ) correction for multiple comparisons to identify markers discriminations between groups. All markers having pvalue $<0.01$ were selected for further tentative annotation process.

Discriminating markers between the three groups identified through multi- and univariate analyses were first screened using a list of known APAP metabolites. The suspect list was build using information already available in the literature and using in silico software tools that predicts small molecule metabolism in mammals (e.g., BioTransformer (Djoumbou-Feunang et al., 2019a)). Identities of these expected APAP metabolites were determined from accurate mass, isotopic fit, fragmentation data obtained from SWATH acquisition and from comparison with standard compounds when available or spectra available in online libraries or the literature. In order to provide confidence in metabolite identification, we supply confidence levels based on recommendations made by Schymanski et al. (2014) (see Supp. Table S6). The structural identities of unknown discriminating markers were determined from their accurate mass, isotopic fit, fragmentation data obtained from SWATH acquisition. These parameters were compared with those from online libraries such as PubChem (Kim et al., 2019), ChemSpider (Pence and Williams, 2010), KEGG (Kanehisa, 2002), HMDB (Wishart et al., 2018b), Metlin (Guijas et al., 2018), T3DB (Wishart et al., 2015), MassBank (Horai et al., 2010) or DrugBank (Wishart et al., 2018a). When no experimental spectra were available, MS/MS fragmentation patterns of individual markers were collected and annotation was based on online software such as MetFrag (Ruttkies et al., 2016) and CFM-ID (Djoumbou-Feunang et al., 2019b)) which allow to perform in silico fragmentation of candidate molecules from different databases (e.g., PubChem) and search for matched against mass to charge values. When possible, chemical identity was confirmed from accurate mass, isotopic fit and fragmentation data obtained from standard compounds (highest level of confidence). In the end, only annotations with a confidence level higher than $2 \mathrm{~b}$ (i.e., based on MS/MS match with experimental or in silico MS/MS spectra) were reported.

2.1.4.6. Accurate integration of identified and annotated markers. Independent peak integration and normalization were carried out to validate the significance of the discriminated metabolites between groups. All the markers identified were manually re-integrated using the Sciex OS Analytics tool. The integrated peak area of individual markers was normalized to APAP-d4 for plasma or to a corrective factor based on the total peak area measured with MarkerView for urine (this corrective factor was calculated by dividing the total peak area of each sample to that of a reference sample). Prism 8 software (GraphPad Software) was used to generate plots.

\section{Results and discussion}

\subsection{Targeted analyses of APAP in blood plasma and urine}

Results with targeted analyses confirmed the presence of APAP in urine $(0.107 \pm 0.025 \mathrm{mg} / \mathrm{L}$, mean $\pm \mathrm{SEM}, \mathrm{min}=0.002 \mathrm{mg} / \mathrm{L}$, $\max =$ $0.200 \mathrm{mg} / \mathrm{L} ; \mathrm{n}=12$ ) before the intentional APAP dose, similar to concentrations previously observed in the German (Modick et al., 2014) and the Danish populations (Nielsen et al., 2015). As illustrated in Fig. 1, the peak APAP concentration in blood plasma was observed already one hour after APAP intake (mean concentration of $19.2 \mathrm{mg} / \mathrm{L}$ ) in the range of plasma therapeutic concentrations previously measured $(5-25 \mathrm{mg} / \mathrm{L})$ (Schulz et al., 2012). In urine, the peak APAP concentration was observed after $4 \mathrm{~h}$ (mean concentration of $1.83 \mathrm{~g} / \mathrm{L}$ ). After $24 \mathrm{~h}$ and $48 \mathrm{~h}$, median urinary APAP concentrations were $2.7 \%$ (mean concentration of $49.6 \mathrm{mg} / \mathrm{L}$ ) and $0.3 \%$ (median concentration of $5.6 \mathrm{mg} / \mathrm{L}$ ) of the maximum mean urinary concentrations, respectively.

The mean APAP urinary concentrations observed 24-48 h after APAP intake were about 50-450 fold higher than the one observed before APAP intake, but overlap with the environmental APAP concentrations previously observed in urine from the general population among nonusers and non-smokers from 0.95 to $580.4 \mathrm{mg} / \mathrm{L}$, mean $=4.1 \mathrm{mg} / \mathrm{L}$ (Modick et al., 2014), confirming that, in some cases, pharmaceutical APAP use cannot be distinguished from the environmental exposure after $24 \mathrm{~h}$ using present standard targeted methods.

\subsection{Detection of new APAP metabolites in humans using HRMS-based non-targeted analyses}

To gain a deeper insight into APAP metabolism, we next analyzed samples from the longitudinal exposure with a non-targeted HRMSbased method. Discriminating markers between the baseline group (samples collected two days and just prior APAP intake), the $+1-6 h$ group (samples collected from $1 \mathrm{~h}$ up to $6 \mathrm{~h}$ after APAP intake), and the $+1-2$ days group (samples collected in the morning of the subsequent two days after APAP intake) were identified through multi- and univariate analyses. In total, APAP and 14 of its (proposed or confirmed see below) metabolites could be annotated and identified in blood and urine after APAP intake with confidence levels ranging from $2 \mathrm{a}-2 \mathrm{~b}$ (annotation based on MS/MS match with experimental or in silico MS/ MS spectrum) to 1 (confirmation of identification with a standard) according to Schymanski et al. (Schymanski et al., 2014). The list of all APAP metabolites detected in human blood and urine, their respective confidence levels, as well as the metabolic pathway involved are reported in Table 1 (see Table S6 for more information on criteria used for the annotation/identification including the list of MS/MS fragments).

Based on relative peak areas, the main APAP metabolites detected in blood and urine samples collected $+1-6 h$ after APAP intake include direct phase II glucuronide (APAP-Glu) and sulfate (APAP-S) conjugates. APAP-Glu and APAP-S are formed by direct glucuronidation and sulfation of APAP in the liver through the action of sulfotransferases (ST) and 

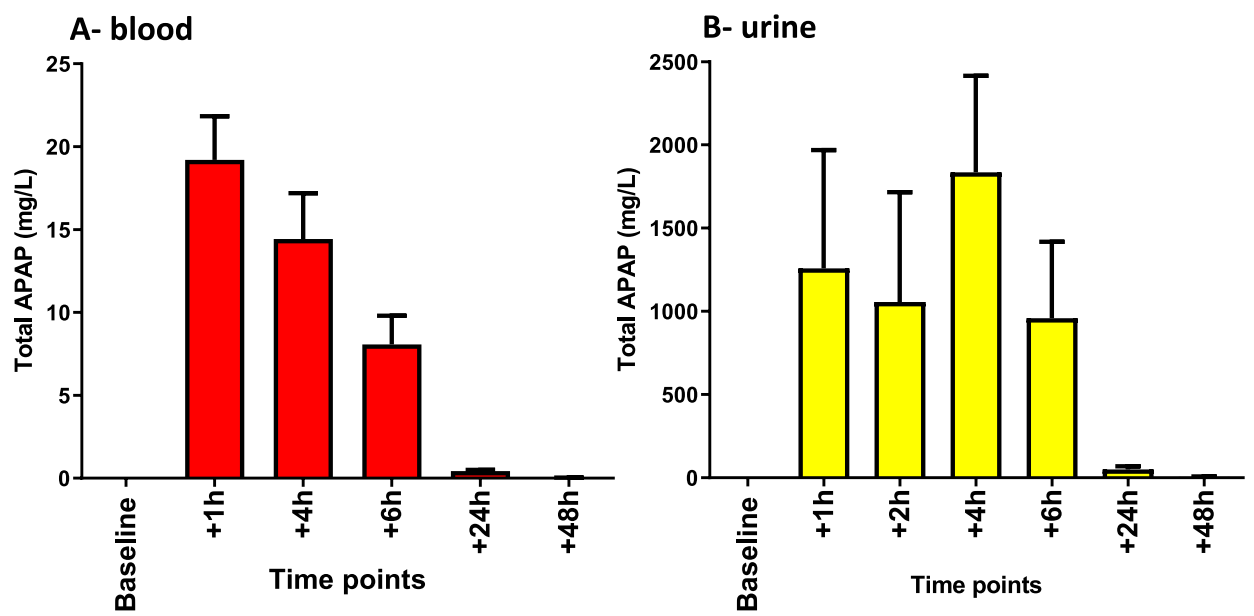

Fig. 1. "Total" APAP concentrations (free APAP, APAP glucuronide and APAP sulfate) after enzymatic deconjugation using LC-MS/MS in (A) blood plasma (mg/L, mean \pm SEM) and (B) urine (mg/L, mean \pm SEM) of the 4 men before APAP intake (baseline, $n=12,0.107 \pm 0.025 \mathrm{mg} / \mathrm{L}$, mean \pm SEM in urine) and at different time points after intake. Urine concentrations are normalized to specific gravity.

Table 1

List of APAP and its metabolites detected in human blood and urine at different time points after APAP intake using high-resolution mass spectrometry. The highest level of evidence used for the annotation and identification of APAP metabolites, their respective pathway and the enzymes involved as well as previous detections in human or mammalians are reported.

\begin{tabular}{|c|c|c|c|c|c|c|}
\hline $\begin{array}{l}\text { List of APAP/metabolites } \\
\text { and associated pathways }\end{array}$ & Formula & $\begin{array}{l}\text { Confid. } \\
\text { Level }^{1}\end{array}$ & $\begin{array}{l}\text { Higest level of evidence for } \\
\text { identification/annotation }\end{array}$ & Main enzymes involved & $\begin{array}{l}\text { Reported in } \\
\text { humans }\end{array}$ & $\begin{array}{l}\text { Reported in } \\
\text { rodents/dogs }\end{array}$ \\
\hline \multicolumn{7}{|l|}{ Parent } \\
\hline \multicolumn{7}{|l|}{ Direct phase II reactions } \\
\hline APAP-S & C8H9NO5S & 2a & $\begin{array}{l}\text { MSMS match with experimental } \\
\text { MSMS spectra from a library }\end{array}$ & STs & Yes & Yes \\
\hline APAP-Glu & C14H17NO8 & 1 & Rt and MSMS match with std & UDPGTs & Yes & Yes \\
\hline \multicolumn{7}{|l|}{ Catechol pathway } \\
\hline 3-OCH 3 -APAP-Glu & C15H19NO9 & 2a & $\begin{array}{l}\text { MSMS match with experimental } \\
\text { MSMS spectra from a library }{ }^{2}\end{array}$ & & Yes & Yes \\
\hline \multicolumn{7}{|l|}{ Mercapturic acid pathway } \\
\hline APAP-Cys & $\mathrm{C} 11 \mathrm{H} 14 \mathrm{~N} 2 \mathrm{O} 4 \mathrm{~S}$ & 1 & Rt and MSMS match with std & $\begin{array}{l}\text { CYP450 (for NAPQI) + GSTs + } \\
\text { GGTs, CGDs + NATs }\end{array}$ & Yes & Yes \\
\hline NAC-APAP & C13H16N2O5S & 1 & Rt and MSMS match with std & & Yes & Yes \\
\hline NAC-O-APAP & C13H16N2O6S & $2 b$ & $\begin{array}{l}\text { MSMS match with in silico MSMS } \\
\text { spectra }^{3}\end{array}$ & & No & no \\
\hline $\mathrm{S}-\mathrm{CH}_{3}-\mathrm{APAP}$ & C9H11NO2S & 1 & Rt and MSMS match with std & & Yes & Yes \\
\hline S-CH - APAP-S & C9H11NO5S2 & 2b & $\begin{array}{l}\text { MSMS match with experimental } \\
\text { MSMS spectra (literature) }\end{array}$ & & No & Yes \\
\hline S-CH ${ }_{3}$-APAP-Glu & C15H19NO8S & $2 b$ & $\begin{array}{l}\text { MSMS match with in silico MSMS } \\
\text { spectra }^{3}\end{array}$ & & No & Yes \\
\hline $\mathrm{SO}-\mathrm{CH}_{3}$-APAP-S & C9H11NO6S2 & $2 b$ & $\begin{array}{l}\text { MSMS match with in silico MSMS } \\
\text { spectra }^{3}\end{array}$ & & No & $\begin{array}{l}\text { Only as } \\
\text { unconjugated }\end{array}$ \\
\hline SO-CH ${ }_{3}$-APAP-Glu & C15H19NO9S & $2 b$ & $\begin{array}{l}\text { MSMS match with in silico MSMS } \\
\text { spectra }^{3}\end{array}$ & & No & $\begin{array}{l}\text { Only as } \\
\text { unconjugated }\end{array}$ \\
\hline
\end{tabular}

STs = sulfotransferases; UDPGTs = uridine diphosphoglucuronyltransferases; GSTs = glutathione $S$-transferases; GGTs = gamma glutamyltransferases, CGDs = cysteinylglycine dipeptidases; NATs = N-acetyltransferases; CS lyases = Cysteine S-conjugate $\beta$-lyases; SMTs = S-methyl-transferases

${ }^{1}$ Schymanski et al., 2014.

${ }^{2}$ Metlin, HMDB.

${ }^{3}$ MetFrag and CFM-ID.

${ }^{4}$ Patterson et al., 2013.

uridine diphosphoglucuronyltransferase (UDPGTs) (Mazaleuskaya et al., 2015). We also detected in this group glutathione-derived APAP metabolites originating from the phase I reactive NAPQI, i.e., APAP-Cys and NAC-APAP. APAP is activated in the liver to form the toxic NAPQI under the mediation of CYP450 enzymes which is then almost immediately detoxified in the liver through the action of the glutathione transferases (Bessems and Vermeulen, 2001). Glutathione conjugates are then biotransformed in the liver as APAP-Cys and then NAC-APAP through the mercapturic acid pathway by the sequential action of the $\gamma$-glutamyltransferases, dipeptidases, and cysteine $S$-conjugate $\mathrm{N}$ - 
acetyltransferase (Hanna and Anders, 2019). Finally, we also detected in this $+1-6 h$ group catechol APAP metabolites originating from another phase I oxidation pathway (i.e., 3-OH-APAP and 3- $\mathrm{OCH}_{3}$-APAP present as non-conjugated and conjugated forms).

Of the 14 metabolites, we also annotated conjugated forms of Smethyl-3-thioacetaminophen (S- $\mathrm{CH}_{3}$-APAP-S and S- $\mathrm{CH}_{3}$-APAP-Glu) and S-methyl-3-thioacetaminophen sulphoxide (SO-CH 3 -APAP-S and SO$\mathrm{CH}_{3}$-APAP-Glu) up to now unreported in humans as conjugated. These 4 conjugated metabolites had previously only been reported in rodents (rat and hamster) and dogs (albeit non-conjugated in case of $\mathrm{SO}-\mathrm{CH}_{3}-$ APAP-S and SO-CH 3 -APAP-Glu; i.e., SO- $\mathrm{CH}_{3}$-APAP) (Gemborys and Mudge, 1981; Hart et al., 1982; Mikov et al., 1988). The two metabolites NAC-O-APAP and SH-APAP-Glu have not at all been detected before in any species to the best of our knowledge. Except for NAC-O-APAP, these conjugated thiomethyl metabolites (i.e., S- $\mathrm{CH}_{3}$-APAP-S, S- $\mathrm{CH}_{3}$-APAPGlu, SO-CH 3 -APAP-S and SO- $\mathrm{CH}_{3}$-APAP-Glu metabolites) were mainly detected in samples collected +1-2 days after APAP intake.

In silico fragmentation software such as MetFrag (Ruttkies et al., 2016) and CFM-ID (Djoumbou-Feunang et al., 2019b) were utilized for computer assisted annotation of S-CH 3 -APAP-S and SO- $\mathrm{CH}_{3}$-APAP-S since many potential candidates were matching the matching the accurate mass of these marker ions within PubChem (e.g., 1337 candidates for $m / z=276.004$ in - ESI mode $[\mathrm{M}-\mathrm{H}]^{-} ; 5 \mathrm{ppm}$ mass tolerance). Further confirmation of the S-CH $-\mathrm{CH}_{3}$-APAP-S annotation were obtained by comparing its experimental MS/MS fragments with those of its nonconjugated form (S- $\mathrm{CH}_{3}$-APAP), which was obtained by injecting the $\mathrm{S}_{-} \mathrm{CH}_{3}$-APAP standard in SWATH mode (Fig. 2). Moreover, the MS/MS fragments of $\mathrm{S}-\mathrm{CH}_{3}$-APAP-S we report are matching those observed experimentally in mice after APAP exposure (Patterson et al., 2013). The other marker with an $m / z$ of 291.9945 in -ESI mode $\left([\mathrm{M}-\mathrm{H}]^{-}\right)$could be annotated as $\mathrm{S}^{-\mathrm{CH}_{3}}$-APAP sulfoxide derivative, i.e., $\mathrm{SO}-\mathrm{CH}_{3}$-APAP-S (see Fig. S6 for MS/MS spectra). Although standards or MS/MS spectra are currently unavailable for most of these metabolites, a high level of confidence could be reached (Schymanski et al., 2014; Sumner et al.,
2007) given that a match was observed with the MS/MS profile of the standard for the unconjugated S- $\mathrm{CH}_{3}$-APAP and that the metabolites are related to the same pathway of elimination of thio metabolites.

\subsection{Relevance of thiomethyl metabolites for monitoring APAP pharmaceutical use}

To understand the relevance of the 4 conjugated thiomethyl metabolites (S- $\mathrm{CH}_{3}$-APAP-S, S-CH $\mathrm{CH}_{3}$-APAP-Glu, SO- $\mathrm{CH}_{3}$-APAP-S and SO$\mathrm{CH}_{3}$-APAP-Glu) and the two previously unreported metabolites NAC-OAPAP and SH-APAP-Glu metabolites to improve human biomonitoring of APAP, we next investigated the relative contributions of APAP metabolites in urine and plasma based on their analytical responses (normalized peak areas) for each time point. We also compared the analytical responses of free APAP and its phase II conjugates, conventionally used in standard APAP biomonitoring methods, to those of catechol and glutathione-derived APAP metabolites. Although these HRMS-based data are not representative of the importance of each metabolite in APAP metabolism in terms of quantity excreted, they do provide valuable information regarding the most sensitive metabolites (in respect to their ionization efficiency) to be used for optimizing APAP monitoring in humans. The relative contributions based on normalized peak area of APAP metabolites are presented in Fig. 3 for both blood and urine.

Of the 6 metabolites previously unreported in humans, only the sulfate thiomethyl metabolites (i.e. S- $\mathrm{CH}_{3}$-APAP-S and $\mathrm{SO}-\mathrm{CH}_{3}$-APAP-S) present significant analytical responses and therefore potential to improve APAP monitoring. Relative contributions of $\mathrm{S}-\mathrm{CH}_{3}$-APAP-Glu, SO-CH 3 -APAP-Glu, NAC-O-APAP and SH-APAP-Glu are not reported on Fig. 3 as they were $<1 \%$ for all time points after APAP intake. In particular, we observed that S- $\mathrm{CH}_{3}$-APAP-S and SO- $\mathrm{CH}_{3}$-APAP-S are major metabolites for the $24 \mathrm{~h}$ and $48 \mathrm{~h}$ time points in blood and urine while the contributions of these S- $\mathrm{CH}_{3}$-APAP-S and SO-CH 3 -APAP-S metabolites are minor before APAP intake (baseline).

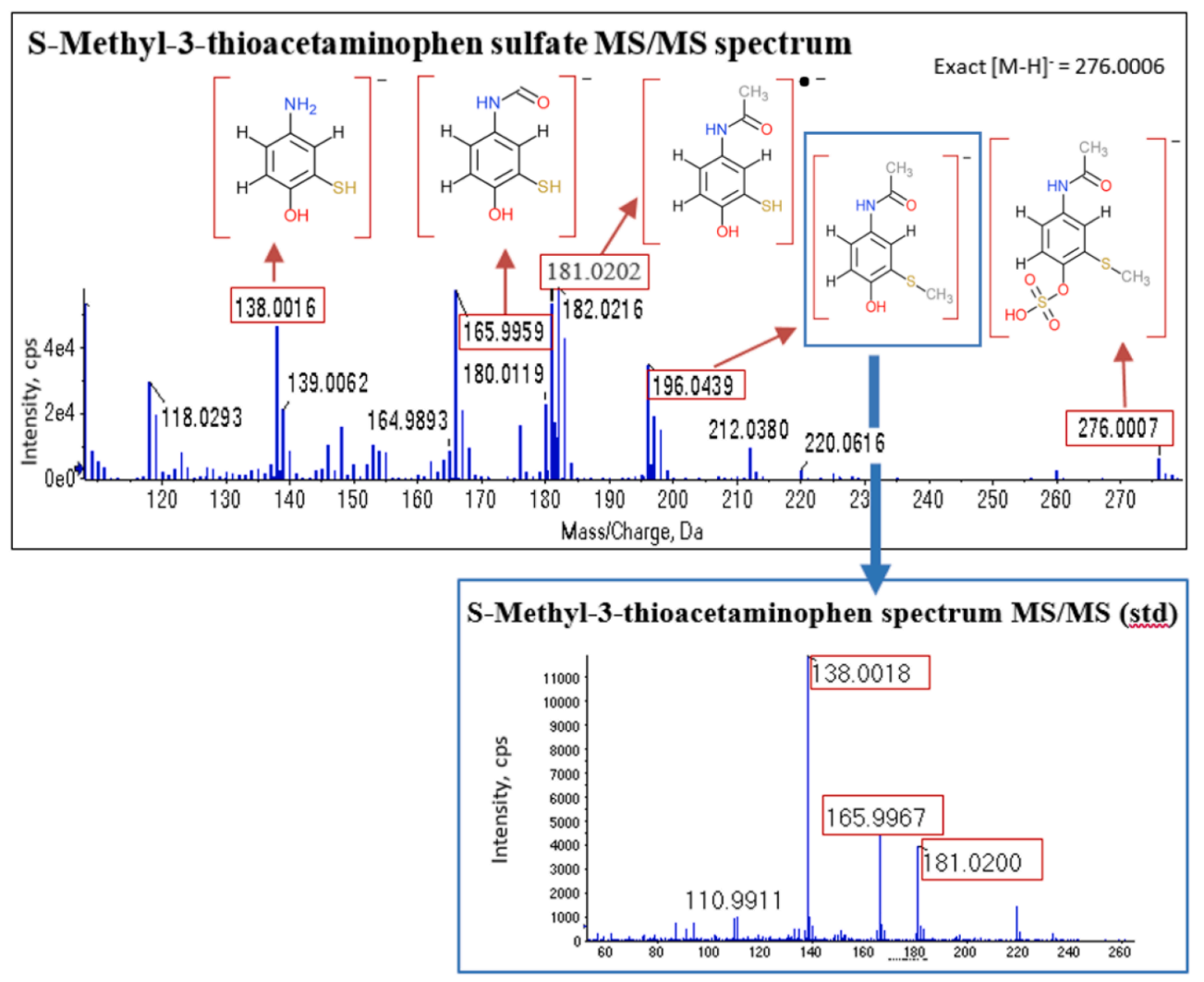

Fig. 2. Experimental MS/MS spectrum of $S$ Methyl-3-thioacetaminophen sulfate from a urine sample $(+24 \mathrm{~h}$ time point $)$ after APAP exposure and MS/MS spectrum of the standard of S-Methyl-3-thioacetaminophen. MS/ MS mass fragmentation information was obtained in SWATH mode using an UHPLC-ESIQTOF-MS in -ESI mode. In silico fragmentation software (i.e., MetFrag and CFM-ID) were utilized for computer assisted identification for $S$-Methyl-3-thioacetaminophen sulfate. Further confirmation of experimental MS/MS fragments could be obtained by injecting the commercially available standard of $S$-Methyl-3-thioacetaminophen. 

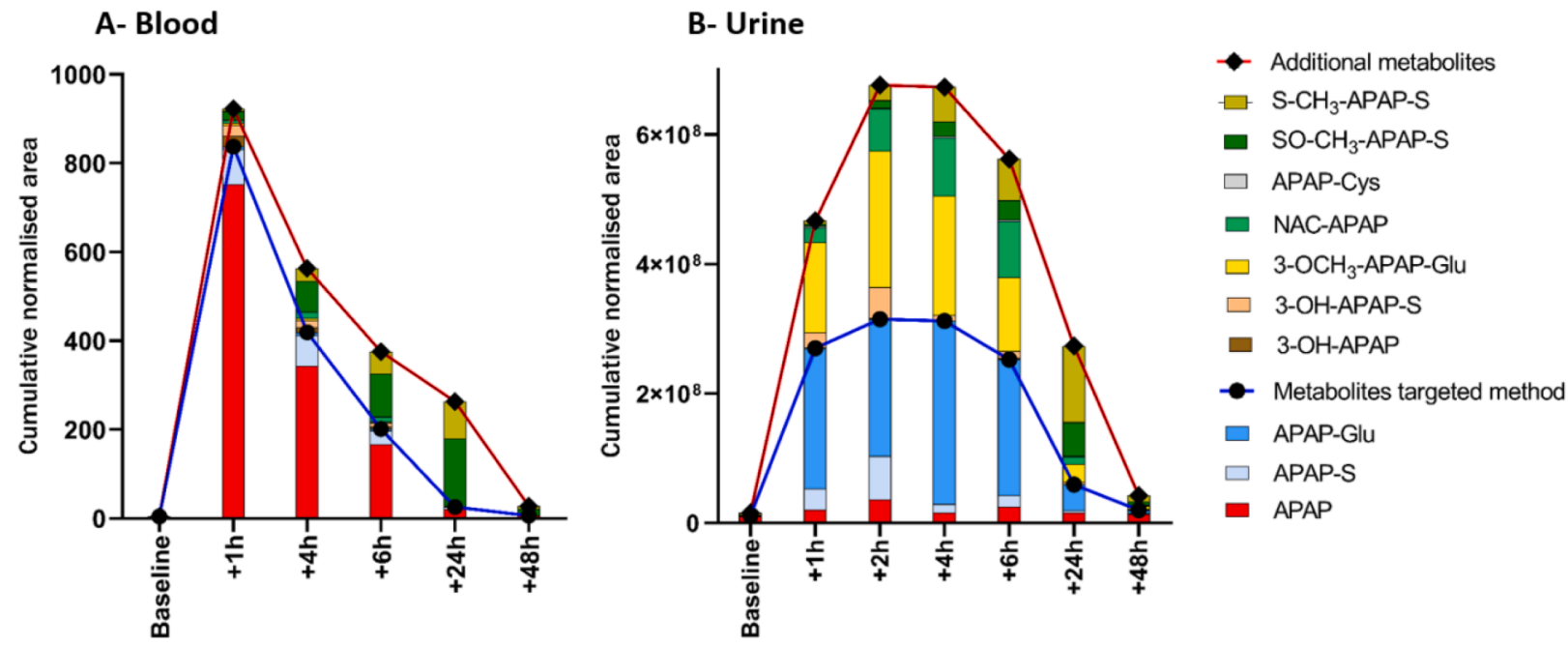

Fig. 3. Analytical responses (represented as cumulative normalized peak areas) of APAP metabolites classically used for targeted methods (i.e., free APAP, APAP-Glu and APAP-S) and the other metabolites detected using the non-targeted analyses in blood (A) and urine (B) of the 4 men. APAP and its metabolites were manually reintegrated using the Sciex OS Analytics tool. The integrated peak area of individual markers was normalized to APAP-d4 for plasma or with a corrective factor based on the total peak area for urine.

More specifically, the relative contribution of the parent APAP was major in blood for the first time points after APAP intake (relative contribution of $44-82 \%$ before $24 \mathrm{~h}$ ) while these of other APAP metabolites such as phase II APAP-S, catechol APAP (OH-APAP and OHAPAP-S), or NAC-APAP were all comprised below $12 \%$ for the same time points. Relative contributions of S- $\mathrm{CH}_{3}$-APAP-S and SO- $\mathrm{CH}_{3}$-APAP$\mathrm{S}$ increased overtime after APAP intake, finally becoming dominant for the $24 \mathrm{~h}$ and $48 \mathrm{~h}$ time points (i.e., $90 \%$ and $78 \%$ at $24 \mathrm{~h}$ and $48 \mathrm{~h}$, respectively, for the sum of both metabolites).

In urine, the metabolites conventionally used for the monitoring of APAP using targeted approach, i.e. free APAP and the phase II conjugates (APAP-S and APAP-Glu) contributed to $45-58 \%$ of the analytical response for the time points comprised between $+1 \mathrm{~h}$ and $6 \mathrm{~h}$. APAP-Glu was the major metabolite for these time points (relative contribution of $31-46 \%$ overall). The other catechol or glutathione-derived metabolites and conjugated thiomethyl metabolites contributed altogether to the remaining $42-55 \%$ of the analytical response from $1 \mathrm{~h}$ up to $6 \mathrm{~h}$ after APAP intake. For these $1-6 \mathrm{~h}$ time points, $3-\mathrm{OCH}_{3}$-APAP-Glu was the major metabolite among these metabolites (relative contribution of $20-31 \%$ overall), followed by NAC-APAP (5- $15 \%$ overall), S- $\mathrm{CH}_{3}$-APAP$\mathrm{S}\left(1-11 \%\right.$ overall) and SO- $\mathrm{CH}_{3}$-APAP-S (0.6-5\% overall). As observed in blood, $\mathrm{S}_{-} \mathrm{CH}_{3}$-APAP-S and SO-CH -APAP-S were major metabolites for the $24 \mathrm{~h}$ and $48 \mathrm{~h}$ time points (relative contribution for the sum of both metabolites of $36-62 \%$ between $24 \mathrm{~h}$ and $48 \mathrm{~h}$ ).

As mentioned earlier, the relative contributions of APAP metabolites reported here are not fully representative of the importance of each metabolite in APAP metabolism in terms of quantity excreted. APAP is known to be extensively metabolized in the liver and based on literature reviews on APAP pharmacokinetics, it is often considered that free APAP and direct phase II conjugates represent between 65 and $85 \%$ of the quantity excreted (mainly as APAP-Glu) while the other catechol and glutathione-derived metabolites can represent up to $20 \%$ together of the quantity excreted (Bessems and Vermeulen, 2001; Mazaleuskaya et al., 2015). However, based on the high analytical response we observed here for the conjugated catechol $3-\mathrm{OCH}_{3}$-APAP-Glu, we show that this metabolite would be a good candidate to improve the analytical sensitivity of targeted methods based on LC-ESI-MS/MS, which is the standard platform used for APAP monitoring.

Background APAP exposure levels did not seem to be accompanied by significant amounts of the conjugated thiomethyl metabolites, while after pharmaceutical use (high exposure) scenario, they could be determined sensitively more than $48 \mathrm{~h}$ post exposure, suggesting that $\mathrm{S}$ -
$\mathrm{CH}_{3}$-APAP-S and SO-CH 3 -APAP-S have great potential to retrospectively assess pharmaceutical use of APAP in humans. Importantly, $\mathrm{S}_{-} \mathrm{CH}_{3}-$ APAP-S and $\mathrm{SO}-\mathrm{CH}_{3}$-APAP-S present similar frequency of distribution in the four individuals compared to APAP metabolites such as APAP-Glu and $3-\mathrm{OCH}_{3}$-APAP-Glu that show the highest analytical sensitivity (see Fig. S7). They could be detected in all individuals (in both blood and urine) with coefficients of variation (CV) ranging from 54 to $74 \%$ in urine at time points close to their peak times. These CVs are similar to those of APAP-Glu and 3-OCH 3 -APAP-Glu (from 18 to 70\%), suggesting that the use of S- $\mathrm{CH}_{3}$-APAP-S and SO- $\mathrm{CH}_{3}$-APAP-S as relevant markers of APAP intake in human could be generalized.

\subsection{The conjugated thiomethyl metabolites and the thiomethyl shunt pathway}

We next studied the kinetics of formation of individual metabolites by comparing their normalized abundance in plasma and urine for each time point before and after APAP intake. The kinetics of formation of free APAP, phase II metabolites (APAP-Glu and APAP-S), catechol metabolites (3-OCH3-APAP-Glu, OH-APAP-S and OH-APAP), glutathione derived metabolites (NAC-APAP and Cyst-APAP) and thiomethyl metabolites $\left(\mathrm{S}-\mathrm{CH}_{3}\right.$-APAP-S and SO-CH -APAP-S) in both blood and urine are presented in Fig. 4.

Similarly to the results obtained with the targeted methods, we observed that APAP was absorbed quickly in the blood and reached the peak blood concentrations within 60 min after ingestion, as previously observed (Mazaleuskaya et al., 2015). The APAP signal had decreased to less than $50 \% 4 \mathrm{~h}$ after intake before reaching basal levels after 24-48 h. Similar kinetics of formation were observed in blood for the minor APAP catechol metabolites (peak time at $1 \mathrm{~h}$ ) while NAC-APAP peaked at $4 \mathrm{~h}$ instead, which could be explained by the fact that NAC-APAP is formed after a higher number of successive reactions involving phase I reactions, glutathione conjugation and enzyme reactions from the mercapturic acid pathway. Interestingly, $\mathrm{S}-\mathrm{CH}_{3}$-APAP-S and SO- $\mathrm{CH}_{3}$-APAP$\mathrm{S}$ metabolites in general showed delayed appearance with a peak time at $24 \mathrm{~h}$ in blood plasma.

In urine, free APAP, phase II metabolite (APAP-S) and catechol metabolites (3-OCH $-\mathrm{OCH}_{3}$-APA-Glu, OH-APAP-S and OH-APAP), peaked at $2 \mathrm{~h}$. This is different from the targeted results that showed that "total APAP" measured as free APAP, APAP-Glu and APAP-S after enzymatic conjugation peaked at $4 \mathrm{~h}$ in urine instead (even though APAP-Glu taken separately also peaked at $4 \mathrm{~h}$ with the non-targeted analyses). However, 


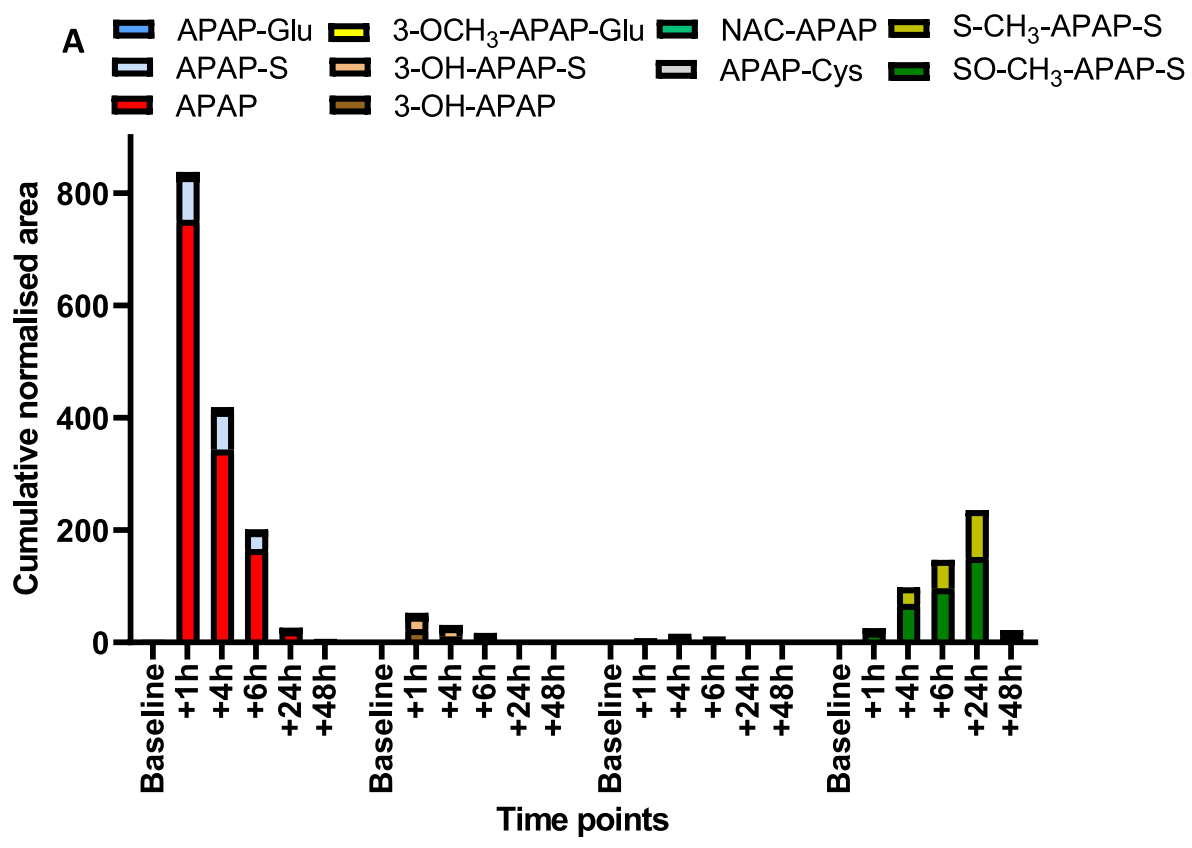

Fig. 4. Kinetics of formation of free APAP, phase II metabolites (APAP-Glu and APAP-S), catechol metabolites $\left(3-\mathrm{OCH}_{3}\right.$-APAP-Glu, OH-APAP-S and OHAPAP), glutathione derived metabolites (NACAPAP and APAP-Cys) and thiomethyl metabolites (S- $\mathrm{CH}_{3}$-APAP-S and SO-CH 3 -APAP-S) detected using the non-targeted screening based on UHPLC-ESIQTOF-MS analyses in (A) blood plasma and (B) urine of the 4 men before intake and at different time points after intake. No significant shift (i.e. below $20 \%$ ) in analytical sensitivity was observed during the batch to make this time point comparison for individual metabolites. APAP and its metabolites were manually re-integrated using the Sciex OS Analytics tool. The integrated peak area of individual markers was normalized to APAP-d4 for plasma or with a corrective factor based on the total peak area for urine.

B

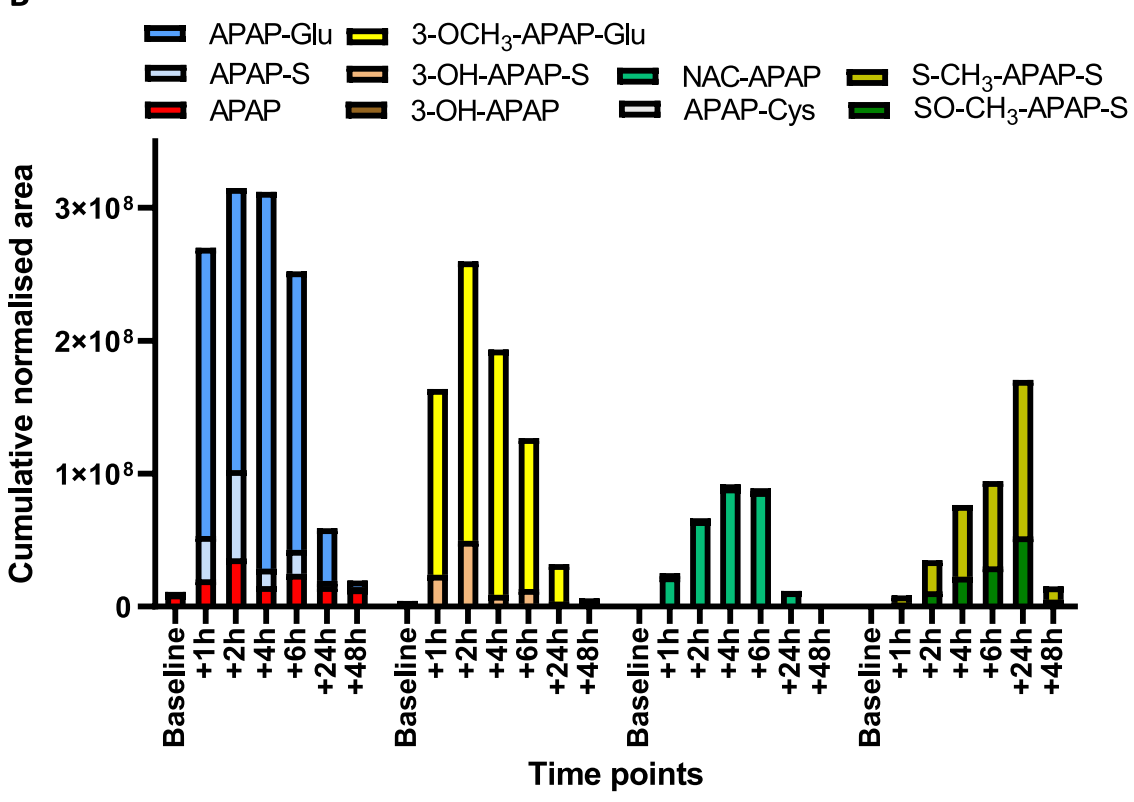

direct comparison of quantitative data from targeted methods and semiquantitative data from HRMS-based methods can be difficult because of the lack of specific correction with proper labelled internal standards. NAC-APAP peaked after the direct phase II conjugates and the catechol metabolites (peak time is at $4 \mathrm{~h}$ ), probably because of the higher number of successive reactions to form the mercapturic acid as mentioned earlier. Like in blood, $\mathrm{S}-\mathrm{CH}_{3}$-APAP-S and SO- $\mathrm{CH}_{3}$-APAP-S metabolites in general showed delayed appearance compared with direct phase II conjugates, catechol metabolites and glutathione-derived metabolites that peaked in the $1-4 \mathrm{~h}$ window.

This delayed appearance suggests that the formation of $\mathrm{S}^{-\mathrm{CH}_{3}}$-APAP$\mathrm{S}$ and $\mathrm{SO}-\mathrm{CH}_{3}$-APAP-S was limited by prior biological steps as opposed to the direct excretion for other metabolites. A similar lag time has been observed in enterohepatic circulation due to the intestinal transit period of metabolites to sites of biotransformation (Roberts et al., 2002). Moreover, the biotransformation that leads to the formation of thiomethyl metabolites have been shown to occur in rodents via the enterohepatic circulation and biliary excretion of the glutathionederived conjugates (i.e., APAP-Cys and NAC-APAP) into the intestine with subsequent microbiotic transformation (Gemborys and Mudge, 1981) (Fig. 5). This transformation includes activity of the cysteine Sconjugate $\beta$-lyase and subsequent methylation involving an active form of methionine (Cooper et al., 2011; Gemborys and Mudge, 1981). Therefore, our data strongly suggests that a thiomethyl shunt pathway (Cooper et al., 2011) that results in formation of the thiomethyl metabolites is also present in humans. In agreement with that, the cysteine conjugate beta-lyase was already previously detected in a majority of gastrointestinal bacteria tested (Larsen, 1985).

Hence, our results show that the conjugated thiomethyl metabolites S- $\mathrm{CH}_{3}$-APAP-S and SO- $\mathrm{CH}_{3}$-APAP-S have the potential to improve APAP biomonitoring in humans since their delayed elimination peaks could extend the window of exposure and thus potentially decrease underestimation or misclassification in epidemiological studies when sampling is performed $>24 \mathrm{~h}$ after intake. 
Tissue metabolism (liver, kidney)

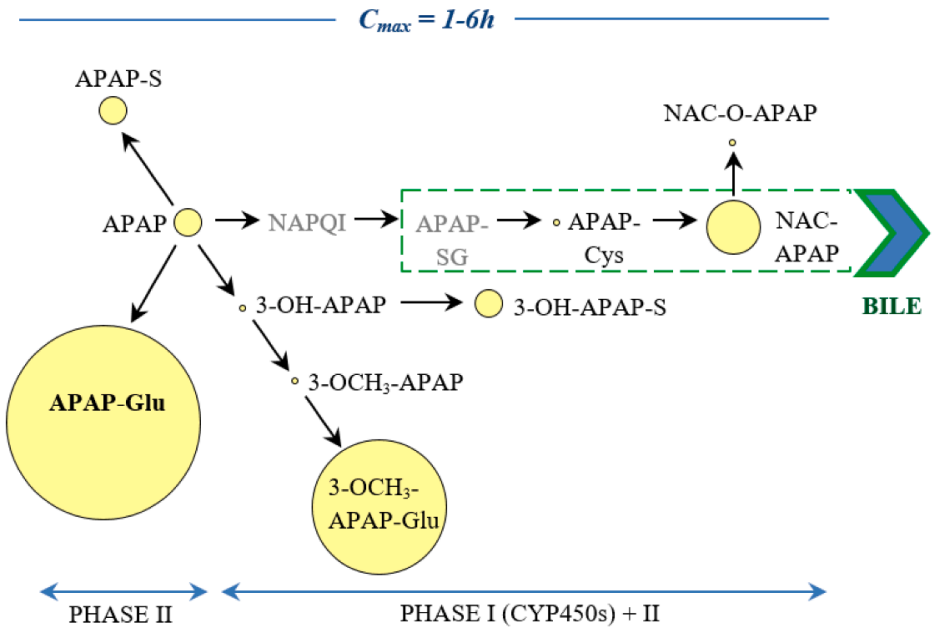

Intestinal metabolism

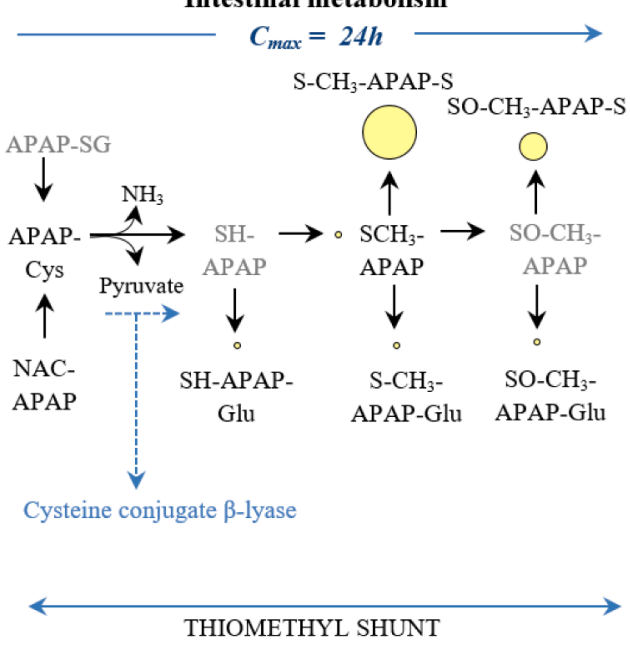

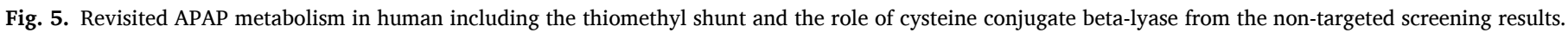
APAP metabolites in grey are known precursors but not detected during this experiment because of their lability or lower levels.

\section{Conclusion}

Reliable biomonitoring methods are essential for proper risk assessment. Estimations of exposure in most existing epidemiological data of APAP have limitations due to potential recall bias or reliance on standard analytical methods for exposure assessment which include metabolites that capture only partially the exposure and/or are too rapidly eliminated. Our data provide a proof-of-concept for HRMS-based non-targeted analyses as a valuable addition to the tool set for the identification of metabolites suitable as exposure biomarkers, complementing HRMS-based suspect screening approaches (e.g., Lessmann et al., 2018) and traditional approaches (e.g., Bury et al., 2019), and thus contributing to the optimization of current standard methods. The findings based on non-targeted analyses after APAP intake suggest that thiomethyl sulfate APAP metabolites and $3-\mathrm{OCH}_{3}$-APAP-Glu should be included in future biomonitoring methods for several reasons. First, the conjugated thiomethyl metabolites have later peak levels and therefore extend the window of exposure decreasing underestimation. Secondly, the data suggest that they provide biomarkers of comparable sensitivity as free APAP and its phase II conjugates. Thirdly, the thiomethyl sulfate APAP metabolites provide information regarding metabolic pathways worth of interest from a toxicological point of view since they are derived from the toxic NAPQI metabolites.

We acknowledge that non-targeted analyses present limitations inherent to the production of semi-quantitative data and therefore the estimates we provide here will require further evaluation based on quantitative measurements and authentic standards. Nevertheless, our quality control data, showing no drift in analytical sensitivity during our batch analyses, provide confidence for the sample to sample comparison made to study the kinetics of appearance of individual metabolites at different time points. The comparison of the relative contributions of APAP metabolites based on their analytical responses is relevant to determine which metabolites can be used to optimize standard targeted methods given that similar platforms (LC-MS) and ion sources (i.e. electrospray ion sources) are utilized for the APAP targeted approach. The standard APAP biomonitoring methods include a deconjugation step using glucuronidases and sulfates. Hence, standard APAP biomonitoring methods could already be upgraded by including $3-\mathrm{OCH}_{3}-$ APAP and $\mathrm{S}-\mathrm{CH}_{3}$-APAP metabolites as these standards are already commercially available. We therefore believe that the limitations in quantitative information value should not overshadow the importance of the identification of the thiomethyl conjugates and their potential to improve the monitoring of non-persistent chemicals such as APAP.
The role of the mercapturate pathway and the cysteine $S$-conjugate beta-lyases in the metabolism of drugs (e.g., methazolamide, cisplatin) have been already demonstrated in mammalians (Cooper et al., 2011). Furthermore, the cysteine $S$-conjugate beta-lyases can play an important key role in the bioactivation of halogenated alkenes (some of which are environmental contaminants produced on an industrial scale), and if the sulfur-containing fragment is reactive, the parent cysteine S-conjugate may be toxic, particularly to kidney mitochondria (Cooper et al., 2011; Cooper and Pinto, 2006). Despite its biological importance, there are surprisingly few data regarding identities of exogenous chemicals detoxified using the mercapturate and thiomethyl shunt pathway. Determining to which extent this happens could improve biomonitoring analytical methods by including more relevant metabolites and thus reduce potential serious underestimations for the reason stated above for APAP. This effort will require a coordinated approach between experts working with both targeted and untargeted approaches and the synthesis of standards for individual chemicals and their metabolites for accurate measurements. Analytical methods based on HRMS are still under development and are not mature enough to replace targeted methods in large biomonitoring programs where accurate quantitative data are needed. However, we advocate for their use as discovery-based approach, as demonstrated here, to update targeted methods with the best metabolites in order to provide the most comprehensive view of the exposure for proper risk assessment for APAP and other similar nonpersistent chemicals.

Author's contribution

$\mathrm{AD}$ contributed to the study design, data collection, data analysis, data interpretation, figures and tables editing, drafting and text revision; JC contributed to the data collection, data analysis, figures and tables editing, text revision; TL contributed to the data analysis, text revision; RAS contributed to the data collection, data analysis, text revision; MDD contributed to the study design, sample collection, text revision; BS contributed to the study design, sample collection; DB contributed to data collection, data analysis, data interpretation, and text revision; HMK contributed to data analysis, data interpretation, figures and tables editing, drafting and text revision; BJ contributed to figures and tables editing, drafting and text revision; DMK contributed to the study design, data collection, data analysis, data interpretation, figures and tables editing, drafting and text revision.

\section{Declaration of Competing Interest}

The authors declare that they have no known competing financial 
interests or personal relationships that could have appeared to influence the work reported in this paper.

\section{Acknowledgments}

We declare no competing interests. We thank Shanna Swan for her editorial comments and Romain Letourneur and Claudia Pälmke for technical assistance. The study was supported by the Candys Foundation and a research chair of excellence (2016-52/IdeX University of Sorbonne Paris Cité) awarded to AD.

\section{Appendix A. Supplementary material}

Supplementary data to this article can be found online at https://doi. org/10.1016/j.envint.2021.106388.

\section{References}

Adusumilli, R., Mallick, P., 2017. Data conversion with ProteoWizard msConvert. Methods Mol. Biol. 1550, 339-368.

Albert, O., Desdoits-Lethimonier, C., Lesne, L., Legrand, A., Guille, F., Bensalah, K., Dejucq-Rainsford, N., Jegou, B., 2013. Paracetamol, aspirin and indomethacin display endocrine disrupting properties in the adult human testis in vitro. Hum. Reprod. 28, 1890-1898.

Bessems, J.G., Vermeulen, N.P., 2001. Paracetamol (acetaminophen)-induced toxicity: molecular and biochemical mechanisms, analogues and protective approaches. Crit Rev. Toxicol. 31, 55-138.

Bornehag, C.G., Reichenberg, A., Hallerback, M.U., Wikstrom, S., Koch, H.M., Jonsson, B. A., Swan, S.H., 2018. Prenatal exposure to acetaminophen and children's language development at 30 months. Eur. Psychiatry 51, 98-103.

Bury, D., Modick-Biermann, H., Leibold, E., Brüning, T., Koch, H.M., 2019. Urinary metabolites of the UV filter octocrylene in humans as biomarkers of exposure. Arch. Toxicol. 93, 1227-1238.

Chetwynd, A.J., Abdul-Sada, A., Hill, E.M., 2015. Solid-phase extraction and nanoflow liquid chromatography-nanoelectrospray ionization mass spectrometry for improved global urine metabolomics. Anal. Chem. 87, 1158-1165.

Cooper, A.J., Krasnikov, B.F., Niatsetskaya, Z.V., Pinto, J.T., Callery, P.S., Villar, M.T., Artigues, A., Bruschi, S.A., 2011. Cysteine S-conjugate beta-lyases: important roles in the metabolism of naturally occurring sulfur and selenium-containing compounds, xenobiotics and anticancer agents. Amino Acids 41, 7-27.

Cooper, A.J., Pinto, J.T., 2006. Cysteine S-conjugate beta-lyases. Amino Acids 30, 1-15.

David, A., Abdul-Sada, A., Lange, A., Tyler, C.R., Hill, E.M., 2014. A new approach for plasma (xeno)metabolomics based on solid-phase extraction and nanoflow liquid chromatography-nanoelectrospray ionisation mass spectrometry. J. Chromatogr. A 1365, 72-85.

David, A., Lange, A., Abdul-Sada, A., Tyler, C.R., Hill, E.M., 2017. Disruption of the prostaglandin metabolome and characterization of the pharmaceutical exposome in fish exposed to wastewater treatment works effluent as revealed by nanoflownanospray mass spectrometry-based metabolomics. Environ. Sci. Technol. 51, 616-624.

Djoumbou-Feunang, Y., Fiamoncini, J, Gil-de-la-Fuente, A., Greiner, R., Manach, C., Wishart, D.S., 2019a. BioTransformer: a comprehensive computational tool for small molecule metabolism prediction and metabolite identification. J. Cheminform. 11, 2.

Djoumbou-Feunang, Y., Pon, A., Karu, N., Zheng, J., Li, C., Arndt, D., Gautam, M., Allen, F., Wishart, D.S., 2019b. CFM-ID 3.0: significantly improved ESI-MS/MS prediction and compound identification. Metabolites 9, 72.

Ferri, F.F., 2016. Ferri's clinical advisor 2017: 5 Books in 1. Elsevier Health Sciences: 1154-1155 ISBN 9780323448383.

Gagnebin, Y., Tonoli, D., Lescuyer, P., Ponte, B., de Seigneux, S., Martin, P.-Y., Schappler, J., Boccard, J., Rudaz, S., 2017. Metabolomic analysis of urine samples by UHPLC-QTOF-MS: Impact of normalization strategies. Anal. Chim. Acta 955, 27-35.

Gemborys, M.W., Mudge, G.H., 1981. Formation and disposition of the minor metabolites of acetaminophen in the hamster. Drug Metab. Dispos. 9, 340-351.

Guijas, C., Montenegro-Burke, J.R., Domingo-Almenara, X., Palermo, A., Warth, B. Hermann, G., Koellensperger, G., Huan, T., Uritboonthai, W., Aisporna, A.E., Wolan, D.W., Spilker, M.E., Benton, H.P., Siuzdak, G., 2018. METLIN: A technology platform for identifying knowns and unknowns. Anal. Chem. 90, 3156-3164.

Hanna, P.E., Anders, M.W., 2019. The mercapturic acid pathway. Crit. Rev. Toxicol. 49, 819-929.

Hart, S.J., Healey, K., Smail, M.C., Calder, I.C., 1982. 3-thiomethylparacetamol sulphate and glucuronide: metabolites of paracetamol and N-hydroxyparacetamol. Xenobiotica 12, 381-386.

Horai, H., Arita, M., Kanaya, S., Nihei, Y., Ikeda, T., Suwa, K., Ojima, Y., Tanaka, K., Tanaka, S., Aoshima, K., Oda, Y., Kakazu, Y., Kusano, M., Tohge, T., Matsuda, F., Sawada, Y., Hirai, M.Y., Nakanishi, H., Ikeda, K., Akimoto, N., Maoka, T., Takahashi, H., Ara, T., Sakurai, N., Suzuki, H., Shibata, D., Neumann, S., Iida, T., Tanaka, K., Funatsu, K., Matsuura, F., Soga, T., Taguchi, R., Saito, K., Nishioka, T., 2010. MassBank: a public repository for sharing mass spectral data for life sciences. J. Mass Spectrom. 45, 703-714.
Hurwitz, J., Sands, S., Davis, E., Nielsen, J., Warholak, T., 2014. Patient knowledge and use of acetaminophen in over-the-counter medications. J. Am. Pharm. Assoc. (2013) 54, 19-26.

Kanehisa, M., 2002. The KEGG database. Novartis Found Symp. 247, 91-101; discussion $101-3,119-28,244-52$

Kim, S., Chen, J., Cheng, T., Gindulyte, A., He, J., He, S., Li, Q., Shoemaker, B.A., Thiessen, P.A., Yu, B., Zaslavsky, L., Zhang, J., Bolton, E.E., 2019. PubChem 2019 update: improved access to chemical data. Nucleic Acids Res. 47, D1102-D1109.

Konkel, L., 2018. Reproductive headache? Investigating acetaminophen as a potential endocrine disruptor. Environ. Health Perspect. 126, 032001.

Kortenkamp, A., 2020. Which chemicals should be grouped together for mixture risk assessments of male reproductive disorders? Mol. Cell. Endocrinol. 499, 110581.

Kortenkamp, A., Koch, H.M., 2020. Refined reference doses and new procedures for phthalate mixture risk assessment focused on male developmental toxicity. Int. J. Hyg. Environ. Health 224, 113428.

Kristensen, D.M., Desdoits-Lethimonier, C., Mackey, A.L., Dalgaard, M.D., De Masi, F, Munkbol, C.H., Styrishave, B., Antignac, J.P., Le Bizec, B., Platel, C., HaySchmidt, A., Jensen, T.K., Lesne, L., Mazaud-Guittot, S., Kristiansen, K., Brunak, S., Kjaer, M., Juul, A., Jegou, B., 2018. Ibuprofen alters human testicular physiology to produce a state of compensated hypogonadism. Proc. Natl. Acad. Sci. USA 115, E715-E724.

Kristensen, D.M., Lesne, L., Le Fol, V., Desdoits-Lethimonier, C., Dejucq-Rainsford, N., Leffers, H., Jegou, B., 2012. Paracetamol (acetaminophen), aspirin (acetylsalicylic acid) and indomethacin are anti-androgenic in the rat foetal testis. Int. J. Androl. 35, 377-384.

Kristensen, D.M., Mazaud-Guittot, S., Gaudriault, P., Lesne, L., Serrano, T., Main, K.M., Jegou, B., 2016. Analgesic use - prevalence, biomonitoring and endocrine and reproductive effects. Nat. Rev. Endocrinol. 12, 381-393.

Larsen, G.L., 1985. Distribution of cysteine conjugate beta-lyase in gastrointestinal bacteria and in the environment. Xenobiotica 15, 199-209.

Lessmann, F., Bury, D., Weiss, T., Hayen, H., Brüning, T., Koch, H.M., 2018. De-novo identification of specific exposure biomarkers of the alternative plasticizer di(2ethylhexyl) terephthalate (DEHTP) after low oral dosage to male volunteers by HPLC-Q-Orbitrap-MS. Biomarkers 23, 196-206.

Mazaleuskaya, L.L., Sangkuhl, K., Thorn, C.F., FitzGerald, G.A., Altman, R.B., Klein, T.E., 2015. PharmGKB summary: pathways of acetaminophen metabolism at the therapeutic versus toxic doses. Pharmacogenet. Genomics 25, 416-426.

Mikov, M., Caldwell, J., Dolphin, C.T., Smith, R.L., 1988. The role of intestinal microflora in the formation of the methylthio adduct metabolites of paracetamol. Studies in neomycin-pretreated and germ-free mice. Biochem. Pharmacol. 37, 1445-1449.

Modick, H., Schütze, A., Pälmke, C., Weiss, T., Brüning, T., Koch, H.M., 2013. Rapid determination of N-acetyl-4-aminophenol (paracetamol) in urine by tandem mass spectrometry coupled with on-line clean-up by two dimensional turbulent flow/ reversed phase liquid chromatography. J. Chromatogr. B 925, 33-39.

Modick, H., Weiss, T., Dierkes, G., Bruning, T., Koch, H.M., 2014. Ubiquitous presence of paracetamol in human urine: sources and implications. Reproduction 147, R105-R117.

Modick, H., Weiss, T., Dierkes, G., Koslitz, S., Kafferlein, H.U., Bruning, T., Koch, H.M., 2016. Human metabolism and excretion kinetics of aniline after a single oral dose. Arch. Toxicol. 90, 1325-1333.

Nielsen, J.K., Modick, H., Morck, T.A., Jensen, J.F., Nielsen, F., Koch, H.M., Knudsen, L. E., 2015. N-acetyl-4-aminophenol (paracetamol) in urine samples of 6-11-year-old Danish school children and their mothers. Int. J. Hyg. Environ. Health 218, 28-33.

Patterson, A.D., Carlson, B.A., Li, F., Bonzo, J.A., Yoo, M.H., Krausz, K.W., Conrad, M., Chen, C., Gonzalez, F.J., Hatfield, D.L., 2013. Disruption of thioredoxin reductase 1 protects mice from acute acetaminophen-induced hepatotoxicity through enhanced NRF2 activity. Chem. Res. Toxicol. 26, 1088-1096.

Pence, H.E., Williams, A., 2010. ChemSpider: an online chemical information resource. J. Chem. Educ. 87, 1123-1124.

Roberts, M.S., Magnusson, B.M., Burczynski, F.J., Weiss, M., 2002. Enterohepatic circulation. Clin. Pharmacokinet. 41, 751-790.

Rohart, F., Gautier, B., Singh, A., Le Cao, K.A., 2017. mixOmics: An R package for 'omics feature selection and multiple data integration. PLoS Comput. Biol. 13, e1005752.

Ruttkies, C., Schymanski, E.L., Wolf, S., Hollender, J., Neumann, S., 2016. MetFrag relaunched: incorporating strategies beyond in silico fragmentation. J. Cheminf. 8, 3 .

Schulz, M., Iwersen-Bergmann, S., Andresen, H., Schmoldt, A., 2012. Therapeutic and toxic blood concentrations of nearly 1,000 drugs and other xenobiotics. Crit. Care 16, R136.

Schymanski, E.L., Jeon, J., Gulde, R., Fenner, K., Ruff, M., Singer, H.P., Hollender, J. 2014. Identifying small molecules via high resolution mass spectrometry: communicating confidence. Environ. Sci. Technol. 48, 2097-2098.

Smith, C.A., Want, E.J., O'Maille, G., Abagyan, R., Siuzdak, G., 2006. XCMS: processing mass spectrometry data for metabolite profiling using nonlinear peak alignment, matching, and identification. Anal. Chem. 78, 779-787.

Sumner, L.W., Amberg, A., Barrett, D., Beale, M.H., Beger, R., Daykin, C.A., Fan, T.W., Fiehn, O., Goodacre, R., Griffin, J.L., Hankemeier, T., Hardy, N., Harnly, J., Higashi, R., Kopka, J., Lane, A.N., Lindon, J.C., Marriott, P., Nicholls, A.W., Reily, M. D., Thaden, J.J., Viant, M.R., 2007. Proposed minimum reporting standards for chemical analysis Chemical Analysis Working Group (CAWG) Metabolomics Standards Initiative (MSI). Metabolomics 3, 211-221.

Want, E.J., Wilson, I.D., Gika, H., Theodoridis, G., Plumb, R.S., Shockcor, J., Holmes, E., Nicholson, J.K., 2010. Global metabolic profiling procedures for urine using UPLC-MS. Nat. Protoc. 5, 1005. 
Wishart, D., Arndt, D., Pon, A., Sajed, T., Guo, A.C., Djoumbou, Y., Knox, C., Wilson, M., Liang, Y., Grant, J., Liu, Y., Goldansaz, S.A., Rappaport, S.M., 2015. T3DB: the toxic exposome database. Nucleic Acids Res. 43, D928-D934.

Wishart, D.S., Feunang, Y.D., Guo, A.C., Lo, E.J., Marcu, A., Grant, J.R., Sajed, T., Johnson, D., Li, C., Sayeeda, Z., Assempour, N., Iynkkaran, I., Liu, Y.,

Maciejewski, A., Gale, N., Wilson, A., Chin, L., Cummings, R., Le, D., Pon, A., Knox, C., Wilson, M., 2018a. DrugBank 5.0: a major update to the DrugBank database for 2018. Nucleic Acids Res. 46, D1074-D1082.
Wishart, D.S., Feunang, Y.D., Marcu, A., Guo, A.C., Liang, K., Vázquez-Fresno, R., Sajed, T., Johnson, D., Li, C., Karu, N., Sayeeda, Z., Lo, E., Assempour, N., Berjanskii, M., Singhal, S., Arndt, D., Liang, Y., Badran, H., Grant, J., Serra-

Cayuela, A., Liu, Y., Mandal, R., Neveu, V., Pon, A., Knox, C., Wilson, M., Manach, C., Scalbert, A., 2018b. HMDB 4.0: the human metabolome database for 2018. Nucleic Acids Res. 46, D608-D617. 